\title{
Dieta de Phyllodactyllus reissi (Sauria:Gekkonidae) en la Zona Reservada de Tumbes, Perú
}

\author{
Diet of Phyllodactyllus reissi (Sauria:Gekkonidae) in the Zona \\ Reservada de Tumbes, Peru
}

\author{
Juan C. Jordán'
}

\begin{abstract}
Departamento de Herpetología, Museo de Historia Natural - Universidad Nacional Mayor de San Marcos. Av. Arenales 1256, Lince, Lima 14. Email:

juan.jordan@gmail.com
\end{abstract}

Presentado: 15/08/2005 Aceptado: $\quad 28 / 03 / 2006$

\begin{abstract}
Resumen
Se describe la dieta de Phyllodactylus reissi, un geckónido nocturno común que se distribuye desde el sur de Ecuador hasta el centro del Perú. Se capturó un total de 15 individuos en la Zona Reservada de Tumbes, en construcciones localizadas en Quebrada Faical, El Caucho y Bocana Murciélago. El análisis del contenido estomacal determinó que $P$. reisii consume seis tipos de presas, principalmente Coleoptera y Blattoptera. El promedio de presas por estómago es de 3,0 con un rango entre 1 y 9 presas. La longitud promedio de las presas fue de $8,98 \mathrm{~mm}$, con un rango entre 1 y $25 \mathrm{~mm}$, y un volumen promedio de $61,97 \mathrm{~mm}^{3}$ y un rango entre 0,13 y $670,8 \mathrm{~mm}^{3}$. La longitud hocico-cloaca y la longitud del hocico no se relacionan significativamente con la longitud y volumen promedio de las presas. Phyllodactylus reissi presenta hábitos generalistas en cuanto a dieta y una tendencia a consumir presas de tamaño mediano en la Zona Reservada de Tumbes.
\end{abstract}

Palabras clave: Phyllodactylus reissi, Gekkonidae, dieta, Zona Reservada de Tumbes, Perú.
Abstract

The purpose of this paper is to describe the diet of Phyllodactylus reissi, a nocturnal common gecko distributed from southern Ecuador to central Perú. A total of 15 individuals were captured inside buildings at Quebrada Faical, El Caucho and Bocana Murciélago in the Zona Reservada de Tumbes. Coleoptera and Blattoptera were the dominant preys from a total of six prey types found in stomachs. The number of prey per stomach were between 1 to 9 , with 3 preys in average; the prey length average was 8,98 $\mathrm{mm}$ with a range of 1 to $25 \mathrm{~mm}$; the prey volume average was $61,97 \mathrm{~mm}^{3}$ with a range of 0,13 to $670,8 \mathrm{~mm}^{3}$. The snout-vent length and snout length were not significantly related with average prey lengths and volumes. Phyllodactylus reissi has generalist habits with respect to diet and shows a tendency to consume medium-sized preys in the Zona Reservada de Tumbes.

Keywords: Phyllodactylus reissi, Gekkonidae, diet, Zona Reservada de Tumbes, Peru.
Phyłlocadyłusreiss es un geckónido nocturno común y abundante en el norte del Perú; su distribución abarca desde el surde Ecuador abarca desde el sur de Ecuador hasta el centro del Perú (Dixon y Huey, 1970; Carrillo e Icochea, 1995), ocupando diversos ecosistemas como el desierto costero peruano, el bosque seco ecuatorial (D ixon and Huey, 1970; Huey, 1979 ) y el bosque tropical del Pacífico, incluido en la Zona Reservada de Tumbes (Tello, 1998). Sin embargo, en Perú existen pocos trabajos sobre la ecología de los Phyllodadylus(Huey, 1979; Carrillo etal., 1990; Werner đal, 1996; Pérez, 2005,). El objetivo del presente estudio es describir, de manera preliminar, la dieta de Phyllodadylus ressi en la Zona Reservada de Tumbes (ZRT).

El trabajo se realizó durante el mes de febrero del 2002 en la ZRT, ubicada en el noroeste del Perú, en las provincias de Zarumilla y Tumbes, en el D epartamento de Tumbes, con un área de 75102 ha, correspondiente a la zona de amortiguamiento de la Reserva de Biosfera del Noroeste (INRENA, 2001). (Actualmente, una porción de la Zona Reservada de Tumbes (56 983.99 has.) ha sido incluida dentro el Parque Nacional Cerros de Amotapey la otra porción (19266.72 has.) constituye la nueva Reserva Nacional de Tumbes (D.S. № 046-2006-AG).
Se realizaron recorridos de búsqueda en transectos predeterminados en los bosques de las siguientes localidades: Q uebrada Faical, El Caucho y Bocana Murciélago y búsquedas en construcciones (puestos de vigilancia del INRENA y policiales fronterizos) entre las 21:00 y 03:00 h. Sólo se encontraron individuos en las paredes de los puestos de vigilancia de Q uebrada Faical, El Caucho y Bocana Murciélago. Los individuos de P. ressi fueron capturados manualmente, sacrificados con una inyección de Ketalar ${ }^{\circledR}$ y sus estómagos fueron extraídos y preservados en alcohol al 70\% en el campo. Los contenidos estomacales fueron analizados con la ayuda de un estereoscopio en el D epartamento de Herpetología, Museo de Historia Natural (Universidad Nacional Mayor de San Marcos). Las presas (artrópodos) fueron identificadas al nivel de Orden. Se registraron las siguientes variables para cada presaidentificada: ancho, largo (precisión de $0,01 \mathrm{~mm}$ ) y volumen $(\mathrm{V}=4 / 3 \pi * \text { largo } / 2 * \text { (ancho/ } 2)^{2}$, Vitt et al., 1995). Las presas fueron categorizadas por rangos de tamaño: 1-5 mm, pequeñas; 6-10 mm, medianas; $10-15 \mathrm{~mm}$., grandes; >15 mm., muy grandes (Huey, 1979). Se midió la longitud hocico-cloaca (LHC) y la longitud de la cabeza (LC) de los individuos colectados de P. ressi. A mbas variables (LHC y LC) 
Tabla 1. Porcentajes de presas consumidas por Phyllodactylus reissien la Zona Reservada de Tumbes.

\begin{tabular}{lcc}
\hline Taxas (presas) & Tumbes (\%) Piura $^{{ }^{\mathbf{a}}} \mathbf{( \% )}$ \\
\hline Blattoptera & 39,3 & 0,0 \\
Orthoptera & 3,6 & 0,0 \\
Hemiptera (inc. Homoptera) & 0,0 & 3,3 \\
Coleoptera & 32,1 & 35,0 \\
Lepidoptera & 10,7 & 0,0 \\
Lepidoptera (larva) & 7,1 & 20,0 \\
Hymenoptera (Formicidae) & 0,0 & 8,3 \\
Diptera & 0,0 & 5,0 \\
Aranae & 7,1 & 5,0 \\
Pseudoscorpiones & 0,0 & 6,7 \\
N (ítems) & 41 & 50 \\
Amplitud de nicho (B) & 5,73 & 5,55 \\
\hline
\end{tabular}

a Huey (1979)

fueron transformadas a $\log _{10}$ para normalizar su distribución (Vitt and Zani, 1997) y empleadas en un análisis de regresión lineal para determinar una probable relación adaptativa entre el tamaño de los geckos y las dimensiones de sus presas.

Se calculó la amplitud del nicho trófico (B) de acuerdo a la fórmula recíproca de Simpson (Pianka, 1973):

$$
\mathrm{B}=1 / \sum_{\mathrm{i}=1}^{\mathrm{n}} \rho_{\mathrm{i}}^{2}
$$

donde i es el uso proporcional del recurso iy n es el número de categorías de presas. El valor de la amplitud de nicho varía de 1 a n, donde valores bajos indican que la dieta se compone de una o pocas categorías de presas, mientras que valores altos indican el uso de más categonías de presas (Pianka, 1973; Vitt \& al, 1994). Se calculó B para el desierto de Sechura (D S) y laZRT incluyendo sólo aquellas presas debidamente identificadas en el presente trabajo y en Huey (1979). Los análisis estadísticos fueron ejecutados con el programa Statistica ${ }^{\circledR}$ v. 5.1 para Windows con un nivel de significancia de $\mathrm{a}=0,05$.

Se capturaron 15 individuos de P. ressi en construcciones localizadas en Q uebrada Faical, El Caucho y Bocana Murciélago en la ZRT. Phyllodadytus reiss consume 6 diferentes tipos de presas, principalmente Coleoptera y Blattoptera (Tabla 1). El



Figura 1. Distribución por longitud $(\mathrm{mm})$ de las presas consumidas por Phyllodactylus reissi en la Zona Reservada de Tumbes



Figura 2. Distribución de volúmenes $\left(\mathrm{mm}^{3}\right)$ de las presas consumidas por Phyllodactylus reissi en la Zona Reservada de Tumbes.

promedio de presas por estómago fue de $3,0 \pm 2,23$, con un rango entre 1 a 9 y una moda de 3 . Las presas tuvieron una longitud promedio de 8,98 $\pm 5,36 \mathrm{~mm}$, con un rango entre $1 \mathrm{y}$ $25 \mathrm{~mm}$, un volumen promedio de $61,97 \pm 108,06 \mathrm{~mm}^{3}$ y un rango entre 0,13 y $670,8 \mathrm{~mm}^{3}$ (Tabla 2, Fig. 1 y 2).

Los coeficientes de regresión indican que el tamaño deP. reissi no se correlaciona significativamente con el número de presas consumidas $\left(\mathrm{R}^{2}=0,067, \mathrm{p}=0,393\right)$ ni con los promedios de volumen y longitud de las mismas $\left(R^{2}=0,002, p=0,869, R^{2}=\right.$ $0,10, p=0,250$; respectivamente). La longitud del hocico tampoco se relaciona significativamente con el promedio del volumen y longitud de las presas $\left(R^{2}=0,057, p=0,094\right.$ y $R^{2}=$ $0,0632, p=0,078$; respectivamente). Al separar las presas por categorías de tamaños, se observa que P. réss consume más presas de tamaño mediano (65,8\%) en laZRT (Fig. 3) mientras que en el D S, consume presas más pequeñas (55\%) (Huey, 1979).

Los tipos y tamaños de las presas consumidas por los individuos de P. reisii en la ZRT difieren de los observados en aquellos del D S (Huey, 1979). Esto podría indicarla ausencia de selectividad por una presa específica, lo que concordaría con la relación no significativa entre la LHC y la LH con respecto al tamaño de las presas (Fig. 1 y 2). Por ejemplo, en el DS, P. ressi

Tabla 2.Descripción estadística de las presas consumidas por Phyllodactylus reissi en la Zona Reservada de Tumbes.

\section{Variable}

Phyllodactyllus reissi (Tumbes)

Número de lagartijas Longitud hocico-ano (SVL)(mm) Número de tipos de presas Número de presas Prom. long. de presas ( $\mathrm{mm})$ Prom. vol. presas $\left(\mathrm{mm}^{3}\right)$
15

$67,95 \pm 8,011(52,2-77,8)$

6

41

$8,98+5,36(1-25)$

$1,97 \pm 108,06(0,13-670,8)$ 


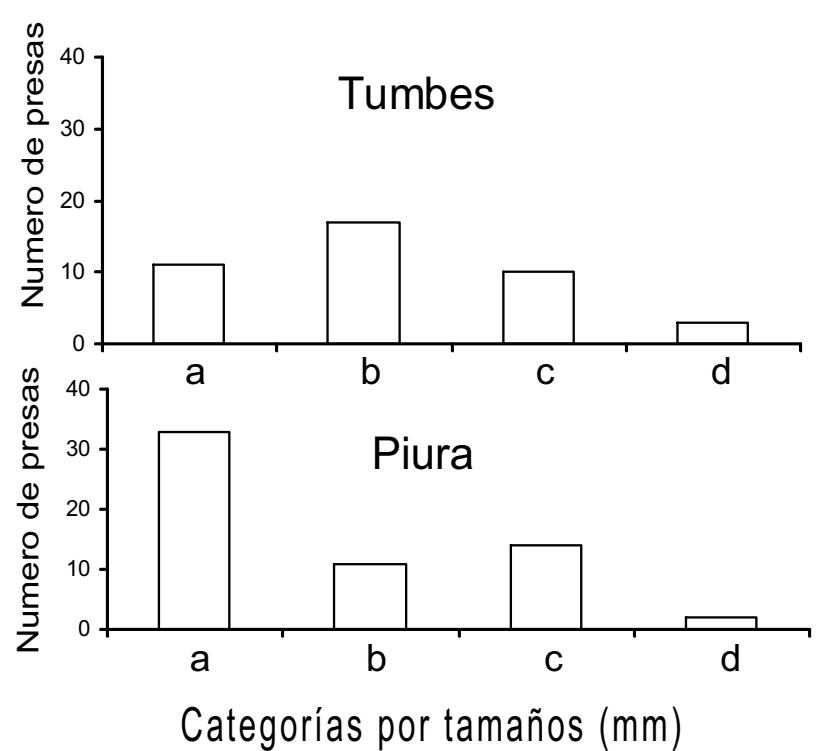

Figura.3. Número de presas consumidas por Phyllodactylus reissi por categorías de tamaño $(\mathrm{mm})$ en la Zona Reservada de Tumbes $(n=41)$ y en el desierto de Sechura $(n=50)$.Categorías: a :1-5mm;b:6$10 \mathrm{~mm}$; c:11-15mm;d:>15mm (ver Huey, 1979).

consume una mayor proporción de presas pequeñas (55\%) mientras que esta misma categoría constituye sólo el 26,8\% de las presas consumidas en la ZRT. Por otro lado Por otro lado, el valor de $B$ para $P$. ressi en la ZRT $(B=5,73)$ es similar al valor obtenido para el $D S(B=5,55)$ sugiriendo que este gecko sería un forrajeador generalista en ambas localidades. Al igual que $\mathrm{P}$. resii, Phyllodadyluslepidgygus $(B=5,06$ ) y otras lagartijas costeras, presentan valores de B similares, considerándoseles también como forrajeadores generalistas (Pérez, 2005).

Sin embargo, ambas poblaciones difieren en algunos taxas consumidos (Tabla 1), debido presumiblemente a la diferencia en la oferta de presas en los distintos microhábitats. Nótese que P. ressi fue capturado en construcciones en la ZRT, donde las cucarachas (Blattoptera) son abundantes, lo cual podría relacionarse con la alta incidencia de éstas en su dieta, mientras que en el DS, P. ressi habita en zonas de rocas grandes y en menor proporción en otros microhábitats (Huey, 1979) asociados con artropofaunas particulares (Tabla 1). En conclusión, Phyllodadytus resii es una lagartija insectívora que presenta hábitos generalistas en cuanto a dieta, consumiendo una amplia variedad de taxas y categorías de tamaños de presas, con tendenciaa consumir aque llas de tamaño mediano en las localidades evaluadas dentro de la ZRT.

\section{Agradecimientos}

A Fernando Cuadros, ex-Jefe de la Reserva de Biosfera del Noroeste por el permiso otorgado para la realización de este trabajo. A José 'El Jefe' Pérez y al revisor anónimo por la revisión crítica del manuscrito. A Jesús Córdova, jefe del D epartamento de Herpetología del Museo de Historia Natural (UNMSM) por permitirme el acceso a sus instalaciones para desarrollar este trabajo.

\section{Literatura citada}

Carrillo, N. y J. Icochea. 1995. Lista taxonómica de los reptiles vivientes del Perú, Publicaciones del Museo de Historia Natural, UNMSM, Serie A, N.o 49, 27 pp.

Carrillo, N., D. Rothenstein. A. Salas and Y. Werner. 1990. Radiation and convergence among desert geckos: Phyllodactylus species resembling both Ptyodactylus and Stenodactylus. Amphibia-Reptilia, 11:1-13

Dixon, J. and R.B. Huey. 1970. Systematic of the lizards of the gekkonidae genus Phyllodactylus of mainland South America. The Natural History Museum of Los Angeles, Contribution in Science. 192:1-78

Huey, R.B. 1979. Parapatry and Niche Complementary of Peruvian Desert Geckos (Phyllodactylus): the Ambiguous Role of Competition. Oecologia, 38: 249-259

INRENA 2001. Estrategia de Conservación y Desarrollo Sostenible de la Reserva de Biosfera del Noroeste 2001-2010. Instituto Nacional de Recursos Naturales (INRENA). Lima-Perú.

Pérez, J.M. 2005. Ecología de duas espécies de lagartos simpátricos em uma formação vegetal de Lomas no Deserto Costeiro Peruano Central. Dissertação do Mestrado. Universidade do Estado de Rio de Janeiro. Rio de Janeiro. Brasil. 78 pp.

Pianka, E. 1973. The structure of lizard communities. Annual Review of Ecology and Systematics 4: 53-74

StatSoft. 1995. STATISTICA for Windows. StatSoft, Inc., Tulsa, Oklahoma, U.S.A.

Tello, G. 1998. Herpetofauna de la Zona Reservada de Tumbes. En: La Zona Reservada de Tumbes: Biodiversidad y Diagnóstico Socioeconómico.W. H. Wust, Ed. The John D. and Catherine C. MacArthur Foundation, PROFONANPE, INRENA. Lima, Perú. Pp. 81-86.

Vitt, L.J. and P. Zani. 1997. Ecology of the nocturnal lizard Thecadactylus rapicauda (Sauria: Gekkonidae) in the Amazon Region. Herpetologica, 53(2): 165-179

Vitt, L.J., P.A. Zani, J.P. Caldwell, and E. Carillo. 1995. Ecology of the lizard Kentropyx pelviceps (Sauria:Teiidae) in lowland rain forest of Ecuador. Canadian Journal of Zoology, 73: 691-703

Werner, Y., N. Carrillo de Espinoza, R. B. Huey, D. Rothenstein, A.W. Salas and F. Videla. 1996. Observations on body temperatures of some neotropical desert geckos (Reptilia: Sauria: Gekkonidae). Cuad. Herp., 10 (1-2): 62-67 
http://sisbib.unmsm.edu.pe/BVRevistas/biologia/biologiaNEW.htm 